\title{
Impact of Immune Checkpoint Inhibitors with or without a Combination of Tyrosine Kinase Inhibitors on Organ-Specific Efficacy and Macrovascular Invasion in Advanced Hepatocellular Carcinoma
}

\author{
Hsin-Yu Kuo a, Nai-Jung Chiang ${ }^{a, b, e}$ Chiao-Hsiung Chuang ${ }^{b}$ \\ Chiung-Yu Chen $^{\mathrm{b}} \quad$ I-Chin Wu $^{\mathrm{b}}$ Ting-Tsung Chang ${ }^{\mathrm{b}}$ Hong-Ming Tsai ${ }^{\mathrm{c}}$ \\ Yih-Jyh Lin ${ }^{d}$
}

${ }^{a}$ Institute of Clinical Medicine, College of Medicine, National Cheng Kung University, Tainan, Taiwan; ${ }^{\text {b Department }}$ of Internal Medicine, National Cheng Kung University Hospital, College of Medicine, National Cheng Kung University, Tainan, Taiwan; ${ }^{C}$ Department of Diagnostic Radiology, National Cheng Kung University Hospital, College of Medicine, National Cheng Kung University, Tainan, Taiwan; ${ }^{\mathrm{d}}$ Department of Surgery, National Cheng Kung

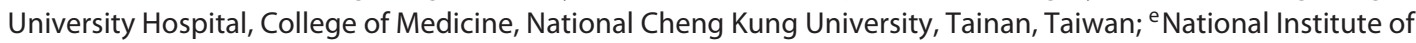
Cancer Research, National Health Research Institutes, Tainan, Taiwan

\section{Keywords}

Hepatocellular carcinoma $\cdot$ Immunotherapy $\cdot$ Immune checkpoint inhibitors · Organ-specific response

\begin{abstract}
Introduction: The tumor microenvironments of different organs often differ and thus may affect the immunotherapy response. Objective: This study elucidated that the efficacy of programmed cell death protein-1 (PD-1) inhibitors varies across different metastatic sites among individuals with advanced hepatocellular carcinoma (HCC). Methods: We retrospectively analyzed treatment outcomes in advanced HCC patients receiving PD-1 inhibitors with or without a combination of tyrosine kinase inhibitors (TKIs). Both the overall response rate (ORR) and organ-specific response rate (OSRR) were assessed using Response Evaluation Criteria in Solid Tumors 1.1 criteria. A survival analysis and its predictors were determined using a multivariate analysis. Results: We analyzed 42 advanced HCC patients (median age: 58.0 years; $78.6 \%$ males). Thirty (71.4\%) patients were sorafenib-experi-
\end{abstract}

enced and 27 (64.3\%) were administered a combination of TKIs. The ORR was $14.3 \%$ and the disease control rate was $33.3 \%$. The median overall survival (OS) and progression-free survival (PFS) were 12.0 and 2.9 months, respectively. The OSRRs were $14.7,23.8,28.6$, and $50.0 \%$ for the liver, lungs, lymph nodes, and vascular response, respectively. The multivariate analysis indicated that the vascular response was significantly associated with PFS. ECOG performance status was a significant independent predictor of OS. Conclusions: PD-1 inhibitors improved OS and PFS in advanced HCC patients. Their efficacies varied among the metastatic locations regardless of the combination of TKIs; in particular, a higher response in vascular metastases was correlated with a longer PFS. PD-1 inhibitors may deliver a synergistic benefit in patients undergoing traditional therapy and progression in other organs in vascular responders.

(c) 2020 S. Karger AG, Basel

Hsin-Yu Kuo and Nai-Jung Chiang are co-first authors.

Hong-Ming Tsai and Yih-Jyh Lin contributed equally to this work. 


\section{Introduction}

Immunotherapy using immune checkpoint inhibitors (ICIs), e.g., programmed cell death protein-1 (PD-1) inhibitors, has recently emerged as a second-line management option for hepatocellular carcinoma (HCC) patients after failure of the first-line agent, sorafenib [1]. Recently, combination therapy comprising ICIs and molecular-targeted drugs has been applied in clinical trials and shows a potential synergic effect and promising preliminary efficacy results in advanced HCC $[2,3]$.

However, responses to immunotherapy are not universal and may be determined by the tumor microenvironment, which may alter immunotherapy effectiveness based on metastasis locations and differences in immune cell content [4]. Previous studies in melanoma and nonsmall cell lung cancer patients have demonstrated that treatment efficacy varies according to the location of the organs involved and the occurrence of immune cells in the metastatic tumors [5]. Response patterns to immunotherapies are clinically important because they can provide further information regarding clinical treatment practices and may help differentiate individuals with oligoprogressive disease who may require additional locoregional or combination therapies [6]. Thus, considering the increasing ICI literature related to HCC treatment, concrete data on tumor response to immunotherapies for different organ sites in HCC patients in clinical settings are unavailable.

Furthermore, advanced HCC patients usually develop macrovascular invasion through direct tumor invasion or metastasis [7]. Tumor invasion of the hepatic vasculature system influences the spread of intrahepatic tumors and manifests as derangement in liver function. Therefore, HCC patients complicated by macrovascular invasion tend to have very poor prognoses. In patients who did not receive any treatment, the median survival time was only 2-3 months [8]. Management options are limited, and survival benefits appear to be unsatisfactory [1, 7-9]. No randomized clinical trials have yet assessed the treatment outcomes of this population. Additionally, little is known about the treatment efficacy of ICIs in vascular involvement in advanced HCC patients.

The different tumor microenvironments of various organs may influence the treatment response of immunotherapy. Additionally, there are no definitive and effective treatment options for advanced HCC patients with macrovascular invasion or extrahepatic spread. We therefore assessed the variations in the tumor response to PD-1 inhibitors with or without a combination of tyrosine kinase inhibitors (TKIs) among different organ sites and the prognostic factors related to treatment outcomes.

\section{Materials and Methods}

\section{Patient Population}

Fifty-eight advanced HCC patients received immunotherapy with or without a combination of TKIs between November 2016 and January 2019 at the National Cheng Kung University Hospital, Tainan, Taiwan. The patients' clinical data were retrieved from the hospital's electronic database. All patients were treated with at least one infusion of PD-1 inhibitors using the standard dose of $3 \mathrm{mg} / \mathrm{kg}$ of nivolumab biweekly or $200 \mathrm{mg}$ of pembrolizumab every 3 weeks, alone or in combination with TKIs. Only patients with subsequent image studies available for response evaluation were enrolled. Data of 9 patients who died prior to their first radiographic imaging assessment were excluded. Among the remaining 49 patients, 7 were excluded (5 with incomplete planned radiographic evaluation and 2 who defaulted from treatment). Thus, 42 patients met the enrollment criteria and were analyzed (Fig. 1).

HCC diagnosis was based on tissue histology or typical radiographic findings. The presence and extent of vascular invasion were diagnosed based on characteristic findings using multiphase dynamic computed tomography (CT) or magnetic resonance imaging (MRI) [10]. No patients had received anticoagulation agents for vascular tumor thrombi.

\section{Radiological Analyses}

Serial CT or contrast-enhanced dynamic MRI, in accordance with the Response Evaluation Criteria in Solid Tumors (RECIST) version 1.1 and modified RECIST (mRECIST), were used to assess the overall response rate (ORR) [11, 12]. All measurements were conducted by two skilled radiologists using the axial image data according to the RECIST 1.1 and mRECIST. For organ-specific response criteria, modified from RECIST 1.1, a maximum of two measurable target lesions per organ was allowed to evaluate the response to immunotherapies for tumors of individual organ systems [13].

The ORR was defined as the percentage of patients with complete remission (CR) or partial response $(\mathrm{PR})$ as the best overall response; the organ-specific response rate (OSRR) was defined as the percentage of patients with $\mathrm{CR}$ or $\mathrm{PR}$ as the best response of the target lesions in a specific organ. The disease control rate (DCR) was defined as the percentage of patients with at least CR, $\mathrm{PR}$, or stable disease (SD) as the best overall response. The organspecific DCR (OSDCR) was defined as the percentage of patients with at least $\mathrm{CR}, \mathrm{PR}$, or $\mathrm{SD}$ as the best organ-specific response.

For vascular invasion, the largest perpendicular diameters of the tumor thrombi, irrespective of the vascular site, were measured. This was compared with the recorded basal value [14]. The product of the maximum of two measurable vascular lesions was used to evaluate the response to immunotherapy. Complete disappearance of the tumor thrombus was defined as a CR, whereas at least a $30 \%$ decrease in the sum of the diameters of the thrombus was defined as a PR; a reduction of $<30 \%$ or an increase of $<20 \%$ was defined as SD, and an increase of $\geq 20 \%$ in the sum of the diameters was defined as PD.

\section{Statistical Analysis}

Survival curves were calculated using the Kaplan-Meier method and compared with the log-rank test. Overall survival (OS) was calculated from the start of the PD-1 inhibitor treatment until death. Progression-free survival (PFS) was calculated from the treatment start until tumor progression according to RECIST or death from any cause, whichever came first. All univariate and multivariate analyses were performed using a Cox proportional 


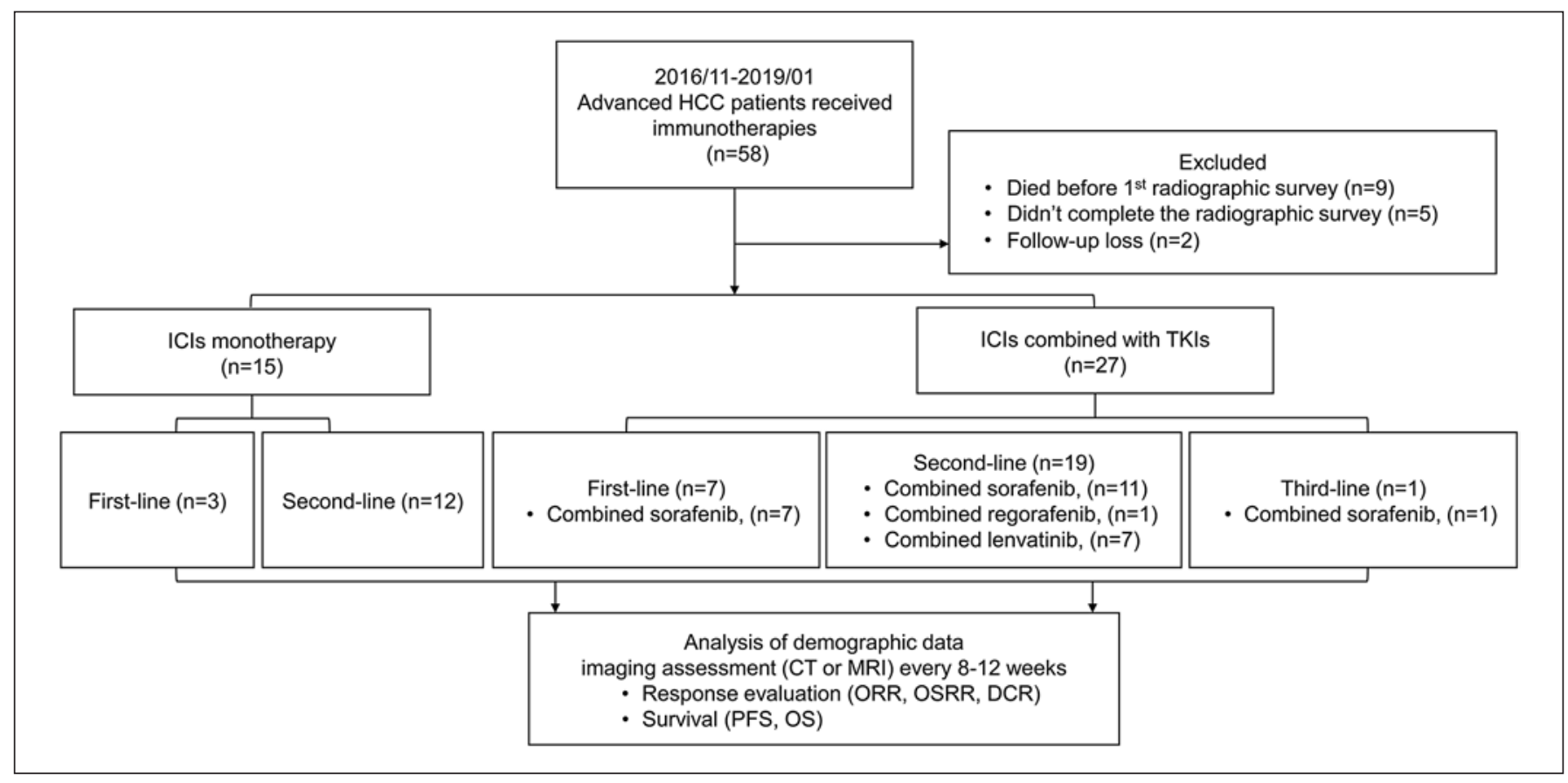

Fig. 1. The proposed study algorithm for the inclusion of subjects. HCC, hepatocellular carcinoma; TKI, tyrosine kinase inhibitor; CT, computed tomography; MRI, magnetic resonance imaging; ORR, overall response rate; OSRR, organ-specific response rate; DCR, disease control rate; PFS, progression-free survival; OS, overall survival.

hazards model. Statistically significant variables $(p<0.05)$ in the univariate analysis were chosen for inclusion in the multivariate analysis. $p<0.05$ was considered statistically significant. All analyses were conducted using the SAS statistical package (v. 9.4 for Windows; SAS Institute, Cary, NC, USA).

\section{Results}

\section{Baseline Characteristics}

Overall, 42 patients were analyzed with a median follow-up of 4.6 months (IQR, 3.1-10.2 months) (Table 1). The study included 33 male and 9 female patients (median age, 58.0 years). The most common etiology of liver disease among the patients was hepatitis B virus (78.4\%). The ECOG performance status score was 0 $(n=28), 1(n=11)$, or $2(n=3)$. All patients had BCLC stage $\mathrm{C}$ tumors. Most of the patients had prior sorafenib experience $(71.4 \%)$ with a median sorafenib treatment of 109 days and were classified as Child-Pugh class A (75.6\%). Moreover, 27 patients had been administered combinations of checkpoint inhibitors with sorafenib $(n=19)$, regorafenib $(n=1)$, or lenvatinib $(n=7)$. In 20 cases (19 second-line and 1 third-line) of progression during previous TKI treatments (19 sorafenib and 1 lenvatinib) that received combination therapies as the systemic second-line or third-line treatment, ICIs were added in 11 patients and a combination of ICIs with a different TKI were initiated in 9 patients. Furthermore,

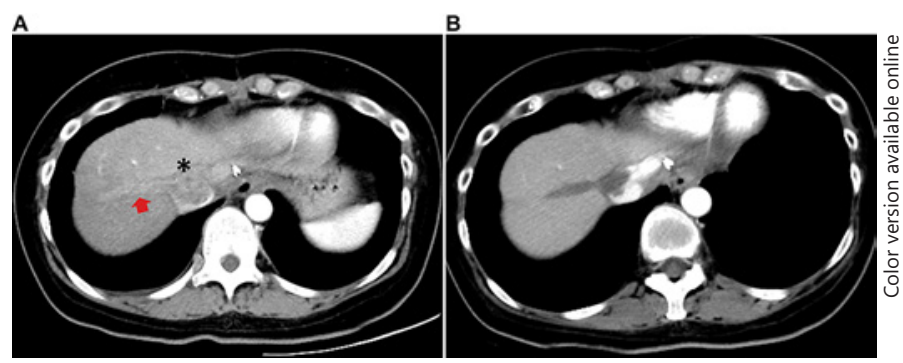

Fig. 2. Serial computed tomography scans in a 52-year-old female with hepatocellular carcinoma (HCC) with an inferior vena cava (IVC) and middle hepatic vein (MHV) tumor thrombus who received PD-1 inhibitor monotherapy. A Before treatment, the thrombus was expanded and enhanced in the $\operatorname{IVC}(*$ in $\mathbf{A})$ and MHV (arrow in A). B Three months after PD-1 inhibitor monotherapy, markedly reduced enhancement and the largest perpendicular diameters of the tumor thrombus over the IVC and MHV were revealed.

$26(61.9 \%)$ patients showed extrahepatic involvement, with extrahepatic lesions most commonly manifested in the lungs ( $n=21,80.8 \%)$. Additionally, 24 (57.1\%) patients had vascular invasion, including the main portal vein $(n=13,54.2 \%)$, first-order branches of the portal vein $(n=3,12.5 \%)$, inferior vena cava (IVC) tumor thrombosis $(n=4,16.7 \%)$, right ventricle $(n=1,4.2 \%)$, and IVC tumor thrombosis plus first-order branches of the portal vein $(n=3,12.5 \%)$. 
Table 1. Patient demographics and baseline clinical characteristics $(\mathrm{n}=42)$

\begin{tabular}{|c|c|}
\hline \multicolumn{2}{|l|}{ Gender } \\
\hline Female & $9(21.4)$ \\
\hline Male & $33(78.6)$ \\
\hline Age, years & $58.0(51.0-65.0)$ \\
\hline$<55$ years & $12(28.6)$ \\
\hline$\geq 55$ years & $30(71.4)$ \\
\hline ECOG & $0.0(0.0-0.5)$ \\
\hline 0 & $28(66.7)$ \\
\hline 1 & $11(26.2)$ \\
\hline 2 & $3(7.1)$ \\
\hline$\alpha$-Fetoprotein, $\mathrm{ng} / \mathrm{mL}^{\mathrm{a}}$ & $172.0(8.2-3,568.0)$ \\
\hline$<400 \mathrm{ng} / \mathrm{mL}$ & $23(59.0)$ \\
\hline$\geq 400 \mathrm{ng} / \mathrm{mL}$ & $16(41.0)$ \\
\hline \multicolumn{2}{|c|}{ Etiology of chronic liver disease } \\
\hline No liver disease & $5(11.9)$ \\
\hline Liver disease present & $37(88.1)$ \\
\hline Chronic hepatitis B & $29(78.4)$ \\
\hline Chronic hepatitis C & $6(16.2)$ \\
\hline Alcoholic hepatitis & $2(5.4)$ \\
\hline \multicolumn{2}{|l|}{ Child-Pugh class ${ }^{\text {b }}$} \\
\hline A & $31(75.6)$ \\
\hline B & $10(24.4)$ \\
\hline \multicolumn{2}{|l|}{ BCLC stage } \\
\hline C & $42(100.0)$ \\
\hline \multicolumn{2}{|l|}{ CLIP } \\
\hline $0-1$ & $22(52.4)$ \\
\hline $2-5$ & $20(47.6)$ \\
\hline \multicolumn{2}{|l|}{ Distant metastases } \\
\hline No & $16(38.1)$ \\
\hline Yes & $26(61.9)$ \\
\hline Lung & $21(80.8)$ \\
\hline Bone & $7(26.9)$ \\
\hline Lymph node & $12(46.2)$ \\
\hline Other ${ }^{\mathrm{c}}$ & $5(19.2)$ \\
\hline \multicolumn{2}{|l|}{ Vascular invasion } \\
\hline No & $18(42.9)$ \\
\hline Yes & $24(57.1)$ \\
\hline \multicolumn{2}{|l|}{ Prior treatment } \\
\hline No & $6(14.3)$ \\
\hline Yes & $36(85.7)$ \\
\hline Surgical resection & $18(50.0)$ \\
\hline TACE & $18(50.0)$ \\
\hline RFA/PEI & $9(25.0)$ \\
\hline HAIC & $13(36.1)$ \\
\hline Sorafenib & $30(83.3)$ \\
\hline \multicolumn{2}{|c|}{ PD-1 inhibitors combined with TKIs } \\
\hline No & $15(35.7)$ \\
\hline Yes & $27(64.3)$ \\
\hline Sorafenib & $19(70.4)$ \\
\hline Regorafenib & $1(3.7)$ \\
\hline Lenvatinib & 7 (25.9) \\
\hline
\end{tabular}

Data are presented as $n(\%)$ or median (IQR) as appropriate. ECOG, Eastern Cooperative Oncology Group performance status; CLIP, Cancer of the Liver Italian Program scoring system; BCLC, Barcelona Clinic Liver Cancer; TACE, transcatheter arterial chemoembolization; RFA, radiofrequency ablation; PEI, percutaneous ethanol injection; TKI, tyrosine kinase inhibitor; HAIC, hepatic arterial infusion chemotherapy; PD-1 inhibitors, programmed cell death protein-1 inhibitors. ${ }^{a} 3$ missing values. ${ }^{b} 1$ missing value. ${ }^{\mathrm{c}} 2$ patients with peritoneal metastases, 2 patients with adrenal involvement, and another patient with diaphragm invasion.

\section{Overall and Organ-Specific Response}

The overall ORR by RECIST and mRECIST were 14.3 and $16.7 \%$, respectively. Table 2 shows the ORR and OSRR stratified by organ.

In primary liver tumors, the OSRR was $14.7 \%(n=5)$ with an OSDCR of $38.2 \%(n=13)$. In pulmonary metastases, the OSRR was $23.8 \%(n=5)$ with an OSDCR of $42.9 \%(n=9)$. Additionally, in lymph node metastases, the OSRR was $28.6 \%(n=2)$ and OSDCR was $71.4 \%$ $(n=5)$.

In the assessment of vascular invasion response (Fig. 2), 1 (4.2\%) patient had CR, 11 (45.8\%) had PR, 5 (20.8\%) had SD, and 7 (29.2\%) had PD (Fig. 3). Of the 17 patients with vascular invasion and $\mathrm{PD}$ as their best overall response, 1 (5.9\%), 6 (35.3\%), and 4 (23.5\%) patients had CR, PR, and SD, respectively, as the best organ-specific response at the vascular sites. However, 6 patients had overall PD and vascular invasion PD simultaneously.

\section{Response according to PD-1 Inhibitors Alone or a}

Combination of TKIs and Systemic Lines of ICIs

The ORR by RECIST for PD-1 inhibitors alone versus PD-1 inhibitors combined with TKIs were 20.0 versus $11.1 \%(p=0.649)$. Because too few patients received ICIs as third-line systemic therapy $(n=1)$, we only compared the efficacy of immunotherapy in patients who received ICIs as first- and second-line systemic therapy. The ORR for PD-1 inhibitors with or without a combination of TKIs serving as first-line versus second-line systemic therapy were 10.0 versus $16.1 \%(p=1.000)$.

\section{Survival and Prognostic Factors}

The median OS of the patients was 12.0 months (95\% confidence interval [CI], 5.3 - not estimable), and the median PFS was 2.9 months (95\% CI, 1.9-4.9) (Fig. 4A, B). The median OS was 10.3 months (95\% CI, 2.1 - not estimable) for PD-1 inhibitors alone and 11.1 months $(95 \%$ CI, 5.3-19.3) for PD-1 inhibitors combined with TKIs $(p=0.415)$. The median organ-specific PFS for vascular responders (i.e., presence of vascular response) was 4.9 months and that for vascular nonresponders was 1.7 months $(p<0.004)$ (Fig. 4C).

Cox regression univariate and multivariate analyses were performed to assess the determinants of PFS (Table 3 ). In the univariate analysis, poor ECOG performance status, advanced CLIP staging, overall response, and vascular response were significantly related to PFS. In the multivariate analysis, favorable prognostic factors for PFS included the presence of overall response and vascular response.

The univariate analysis for predictors of OS showed that poor ECOG performance status, advanced CLIP staging, poor liver function (Child-Pugh B), and vascular invasion were unfavorable prognostic factors contribut- 


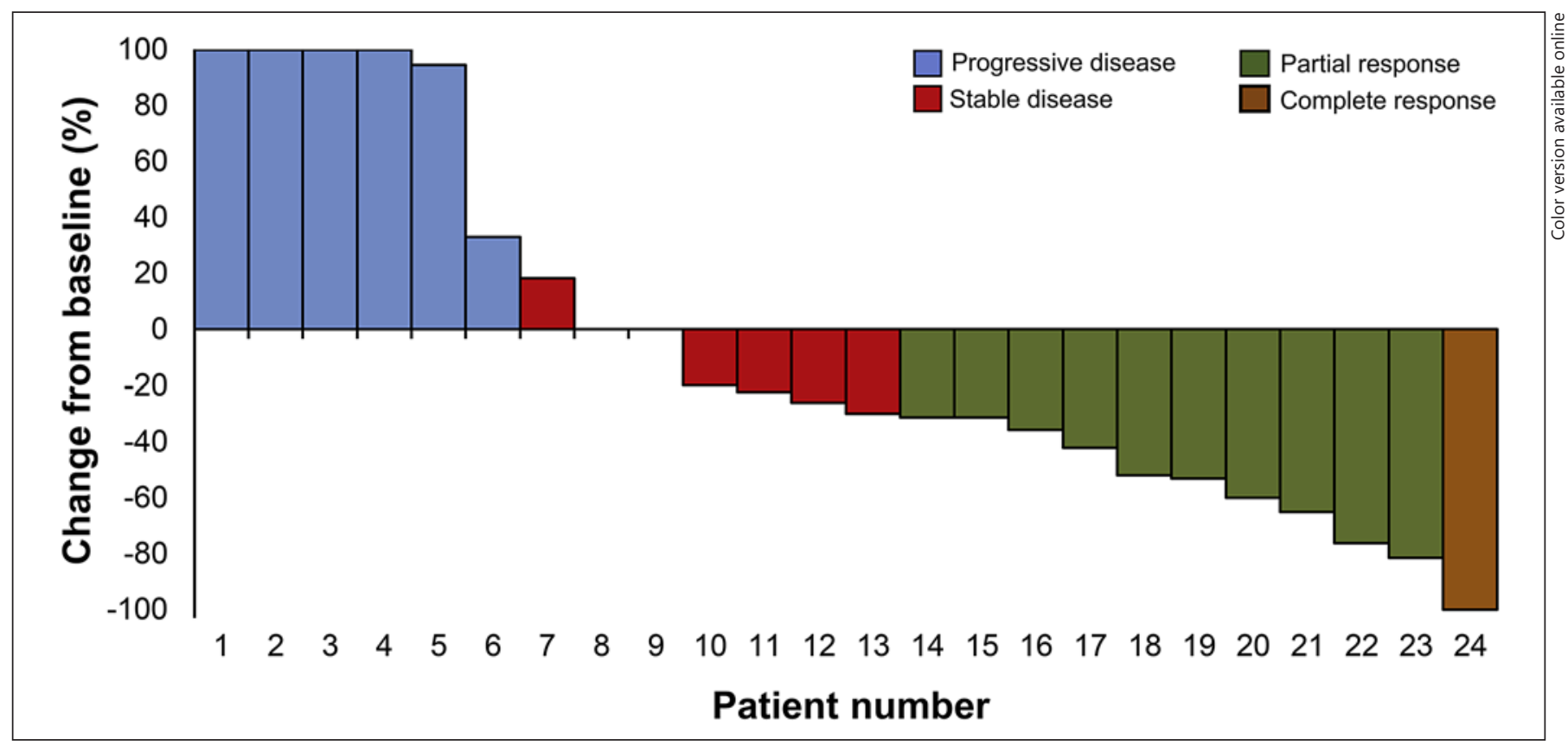

Fig. 3. The best percentage changes in vascular burden $(n=24)$ in patients who were treated with PD-1 inhibitors compared with pretreatment baseline. Numbers along the $x$ axis indicate arbitrarily assigned subject patient numbers from 1 to 24 . The bars indicate the percent change in tumor thrombus burden from baseline.

Table 2. Overall and organ-specific response in the primary tumor, lung, lymph node, and vascular metastases

\begin{tabular}{lcrrrrr}
\hline Response & $\begin{array}{l}\text { Overall } \\
(n=42)\end{array}$ & $\begin{array}{l}\text { Liver } \\
(n=34)\end{array}$ & $\begin{array}{l}\text { Lung } \\
(n=21)\end{array}$ & $\begin{array}{l}\text { LN } \\
(n=7)\end{array}$ & $\begin{array}{l}\text { Vessel } \\
(n=24)\end{array}$ \\
\cline { 2 - 3 } mRECIST & RECIST & & $3(8.8)$ & $3(14.3)$ & $0(0.0)$ & $1(4.2)$ \\
CR & $5(11.9)$ & $4(9.5)$ & $2(5.9)$ & $2(9.5)$ & $2(28.6)$ & $11(45.8)$ \\
PR & $2(4.8)$ & $2(4.8)$ & $8(23.5)$ & $4(19.1)$ & $3(42.9)$ & $5(20.8)$ \\
SD & $9(21.4)$ & $8(19.0)$ & $21(61.8)$ & $12(57.1)$ & $2(28.6)$ & $7(29.2)$ \\
PD & $26(61.9)$ & $28(66.7)$ & $5(14.7)$ & $5(23.8)$ & $2(28.6)$ & $12(50.0)$ \\
ORR & $7(16.7)$ & $6(14.3)$ & $13(38.2)$ & $9(42.9)$ & $5(71.4)$ & $17(70.8)$ \\
DCR & $16(38.1)$ & $14(33.3)$ & & & & \\
\hline
\end{tabular}

Data are presented as $n(\%)$. CR, complete response; PR, partial response; SD, stable disease; PD, progressive disease; ORR, overall response rate; DCR, disease control rate; LN, lymph node; RECIST, Response Evaluation Criteria in Solid Tumors; mRECIST, modified Response Evaluation Criteria in Solid Tumors.

ing to OS (Table 4). The multivariate analysis showed that the ECOG performance status was the most important favorable prognostic indicator of decreased OS.

\section{Discussion}

Immunotherapy with nivolumab and pembrolizumab as second-line agents is now used in the management of advanced HCC patients who are intolerant to or have progressed under approved TKIs [1]. Current knowledge of ICI tumor response patterns in advanced HCC patients based on real-world scenarios is limited. Our study dem- onstrated that tumor response and treatment efficacy to PD-1 inhibitors of advanced HCC differed among various organs, with the best response in vessels and a reduced response in hepatic tumors (OSRR, 50.0 and 14.7\%, respectively). To the best of our knowledge, this is the first population-based cohort study evaluating the response and efficacy of PD-1 inhibitors in the vascular invasion of HCC patients. This study also indicated that vascular response is an independent prognostic factor relative to longer PFS, and ECOG performance status serves as an independent prognostic factor for longer OS. These clinical factors could offer timely and direct information to clinical physicians on whether to continue PD-1 inhibi- 
A

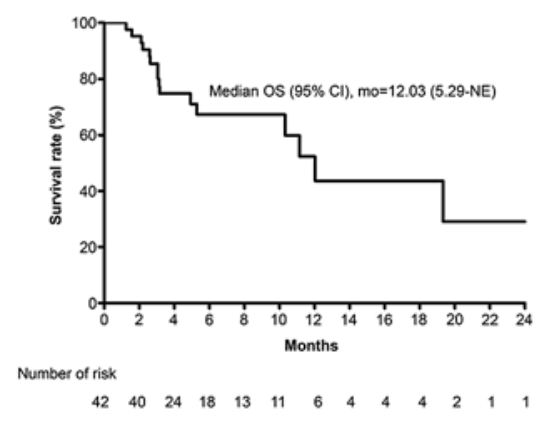

B

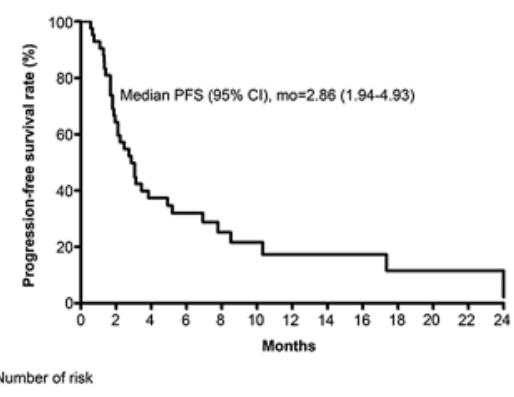

C

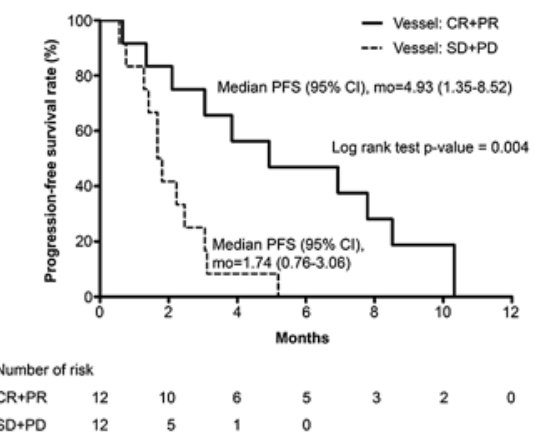

Fig. 4. Kaplan-Meier analysis of overall survival (OS) and progression-free survival (PFS). A The median OS was 12.0 months. B The median PFS was 2.9 months. C The organ-specific PFS of vascular responders (CR+PR) was significantly longer than that of nonresponders $(\mathrm{SD}+\mathrm{PD})$ ( 4.9 vs. 1.7 months, $p=0.004)$. CR, complete response; $\mathrm{PR}$, partial response; $\mathrm{SD}$, stable disease; $\mathrm{PD}$, progression disease; $\mathrm{CI}$, confidence interval; NE, not estimable.

tors and potentially maximize treatment benefits from immunotherapy in HCC.

Recently, two phase 3 trials in patients with advanced HCC both failed to meet the predefined threshold of statistical significance for OS; however, either pembrolizumab or nivolumab demonstrated a favorable ORR (16.9\% for pembrolizumab vs. $2.2 \%$ for placebo; $15.0 \%$ for nivolumab vs. $7 \%$ for sorafenib) $[15,16]$. Another phase 3 trial, IMbrave150, demonstrated significant improvement in both OS (HR, $0.58 ; 95 \% \mathrm{CI}, 0.42-0.79 ; p=$ $0.0006)$ and PFS (HR, 0.59 ; 95\% CI, $0.47-0.76 ; p<0.0001)$ for atezolizumab + bevacizumab versus sorafenib as firstline treatment for advanced HCC patients [3]. In this real-world analysis of consecutive HCC patients treated at a single tertiary center, an ORR of $14.3 \%$, a median PFS of 2.9 months, and an OS of 12.0 months were observed, which were consistent with those in the literature [17]. Previous studies in advanced HCC patients showed a median OS of 6.5 months in those treated with sorafenib and 4.2 months in the placebo group $[18,19]$. These findings support the concept that ICIs are promising in the management of advanced HCC patients and may be considered as a standard or alternative treatment option in clinical practice.

This study demonstrated that the tumor response to PD-1 inhibitors in HCC varied among different organs (Table 2). This diversity of organ-specific response was in line with previous studies and indicates that the immune microenvironments of different organs often differ $[4,5$, $13,20,21]$. Further large cohort-based, long-term followup studies are anticipated to determine whether the response patterns to immunotherapies are clinically relevant.

Approximately $10-40 \%$ of HCC patients have vascular invasion at diagnosis. A previous study indicated that vas- cular invasion was a poor prognostic factor for OS in HCC patients [22]. The management of HCC with vascular invasion remains challenging, and its therapeutic options are limited $[7,8]$. In this study, the response rates of PD-1 inhibitors were higher in vascular invasion (50.0\% ORR) compared with other organ locations. Furthermore, 64.7\% of patients with vascular metastases had ongoing disease control despite overall progression according to RECIST. PFS was also significantly reduced in vascular nonresponders compared with responders (median 1.7 vs. 4.9 months, $p=0.004$ ) (Fig. 4C). According to previous studies, sorafenib showed moderate efficacy in the treatment of vascular invasion with a DCR, PFS, and OS of 33.3\%, 2.0 months, and 3.1 months, respectively $[7,8,18,23]$. Therefore, the efficacy of PD-1 inhibitors in HCC patients with vascular invasion may prevent or halt extensive intrahepatic dissemination of the tumor and preserve hepatic reserves, which may allow further management options such as ablative local treatment to the progressing organ location and continuation of immunotherapies $[24,25]$. This strategy could allow potential benefits to be maximized from the use of immunotherapy for advanced HCC [26].

The multivariate Cox regression analysis indicated that tumor response and vascular response were independent predictors of PFS (Table 3 ). The analysis of the prognostic factors for OS indicated that there were no significant differences in OS between vascular responders and nonresponders (Table 4). Although treatment with PD-1 inhibitors could obtain a vascular response, the efficacy could not be translated into OS benefits. Of 12 patients with a vascular response, 7 (58.3\%) had overall disease progression. Additionally, among those 12 patients, 6 (50\%) did not receive any subsequent anticancer therapy. Thus, in addition to vascular control, the primary tumor or other organ site treatment response must be consid- 
Table 3. Univariate and multivariate analyses of potential prognostic factors for PFS

\begin{tabular}{|c|c|c|c|c|}
\hline & $\begin{array}{l}\text { Crude HR } \\
(95 \% \text { CI })\end{array}$ & $p$ value & $\begin{array}{l}\text { Adjusted } \mathrm{HR}^{\mathrm{a}} \\
(95 \% \mathrm{CI})\end{array}$ & $p$ value \\
\hline \multicolumn{5}{|l|}{ Gender } \\
\hline Female & Ref. & & & \\
\hline Male & $1.19(0.57-2.51)$ & 0.646 & & \\
\hline \multicolumn{5}{|l|}{ Age } \\
\hline$<55$ years & Ref. & & & \\
\hline$\geq 55$ years & $1.26(0.63-2.53)$ & 0.517 & & \\
\hline \multicolumn{5}{|l|}{ ECOG } \\
\hline 0 & Ref. & & Ref. & \\
\hline$\geq 1$ & $2.22(1.16-4.24)$ & 0.016 & $1.43(0.63-3.29)$ & 0.395 \\
\hline \multicolumn{5}{|l|}{ a-Fetoprotein } \\
\hline$<400 \mathrm{ng} / \mathrm{mL}$ & Ref. & & & \\
\hline$\geq 400 \mathrm{ng} / \mathrm{mL}$ & $1.32(0.70-2.49)$ & 0.386 & & \\
\hline \multicolumn{5}{|c|}{ Etiology of chronic liver disease } \\
\hline No liver disease & Ref. & & & \\
\hline Liver disease present & $0.57(0.24-1.37)$ & 0.207 & & \\
\hline \multicolumn{5}{|l|}{ CLIP } \\
\hline $0-1$ & Ref. & & Ref. & \\
\hline $2-5$ & $1.92(1.02-3.63)$ & 0.045 & $0.94(0.41-2.13)$ & 0.877 \\
\hline \multicolumn{5}{|l|}{ Child-Pugh class } \\
\hline A & Ref. & & & \\
\hline $\mathrm{B}$ & $1.61(0.81-3.20)$ & 0.172 & & \\
\hline \multicolumn{5}{|l|}{ Distant metastases } \\
\hline No & Ref. & & & \\
\hline Yes & $0.89(0.48-1.67)$ & 0.725 & & \\
\hline \multicolumn{5}{|l|}{ Vascular invasion } \\
\hline No & Ref. & & & \\
\hline Yes & $1.98(0.93-4.20)$ & 0.075 & & \\
\hline \multicolumn{5}{|l|}{ Previous treatment } \\
\hline No & Ref. & & & \\
\hline Yes & $0.77(0.34-1.76)$ & 0.538 & & \\
\hline Surgical resection & $0.86(0.45-1.63)$ & 0.639 & & \\
\hline TACE & $0.59(0.31-1.12)$ & 0.104 & & \\
\hline RFA/PEI & $1.05(0.50-2.21)$ & 0.901 & & \\
\hline TKIs & $0.76(0.40-1.46)$ & 0.411 & & \\
\hline \multicolumn{5}{|c|}{ PD-1 inhibitors combined with TKIs } \\
\hline No & Ref. & & & \\
\hline Yes & $1.07(0.57-2.01)$ & 0.835 & & \\
\hline \multicolumn{5}{|l|}{ Overall response } \\
\hline No & Ref. & & & \\
\hline Yes & $0.10(0.01-0.74)$ & 0.024 & $0.11(0.02-0.84)$ & 0.033 \\
\hline \multicolumn{5}{|l|}{ Vascular-specific response } \\
\hline No & Ref. & & & \\
\hline Yes & $0.28(0.10-0.76)$ & 0.013 & $0.25(0.09-0.71)$ & 0.009 \\
\hline
\end{tabular}

The overall or organ-specific response was based on the combined number of patients with complete response and partial response. ECOG, Eastern Cooperative Oncology Group performance status; CLIP, Cancer of the Liver Italian Program scoring system; TACE, transcatheter arterial chemoembolization; RFA, radiofrequency ablation; PEI, percutaneous ethanol injection; TKI, tyrosine kinase inhibitor; PD-1 inhibitors, programmed cell death protein-1 inhibitors; Ref., reference level. ${ }^{\text {a }}$ The Cox proportional hazards model.

ered. Furthermore, the diverse treatment modalities after tumor progression might affect survival. Although prognosis in this type of patient is extremely poor, the efficacy of PD-1 inhibitors in HCC patients with vascular invasion may allow further additional treatment options such as combining with other treatment modalities to the progressing organ location.

Distant metastases were not identified as a prognostic factor for patients with HCC, which was in accordance with previous studies [22]. Future long-term follow-up 
Table 4. Predictive factors influencing overall mortality

\begin{tabular}{|c|c|c|c|c|}
\hline & $\begin{array}{l}\text { Crude HR } \\
(95 \% \text { CI })\end{array}$ & $p$ value & $\begin{array}{l}\text { Adjusted HR } \mathrm{HR}^{\mathrm{a}} \\
(95 \% \mathrm{CI})\end{array}$ & $p$ value \\
\hline \multicolumn{5}{|l|}{ Gender } \\
\hline Female & Ref. & & & \\
\hline Male & $1.09(0.43-2.74)$ & 0.861 & & \\
\hline \multicolumn{5}{|l|}{ Age } \\
\hline$<55$ years & Ref. & & & \\
\hline$\geq 55$ years & $1.66(0.62-4.48)$ & 0.313 & & \\
\hline \multicolumn{5}{|l|}{ ECOG } \\
\hline 0 & Ref. & & Ref. & \\
\hline$\geq 1$ & $10.34(3.42-31.25)$ & 0.000 & $11.39(2.16-60.21)$ & 0.004 \\
\hline \multicolumn{5}{|l|}{ a-Fetoprotein } \\
\hline$<400 \mathrm{ng} / \mathrm{mL}$ & Ref. & & & \\
\hline$\geq 400 \mathrm{ng} / \mathrm{mL}$ & $1.48(0.65-3.37)$ & 0.352 & & \\
\hline \multicolumn{5}{|c|}{ Etiology of chronic liver disease } \\
\hline No liver disease & Ref. & & & \\
\hline Liver disease present & $0.69(0.23-2.04)$ & 0.504 & & \\
\hline \multicolumn{5}{|l|}{ CLIP } \\
\hline $0-1$ & Ref. & & Ref. & \\
\hline $2-5$ & $7.08(2.35-21.29)$ & 0.001 & $1.43(0.29-6.95)$ & 0.658 \\
\hline \multicolumn{5}{|l|}{ Child-Pugh class } \\
\hline A & Ref. & & Ref. & \\
\hline $\mathrm{B}$ & $3.07(1.34-7.03)$ & 0.008 & $1.23(0.37-4.05)$ & 0.740 \\
\hline \multicolumn{5}{|l|}{ Distant metastases } \\
\hline No & Ref. & & & \\
\hline Yes & $0.56(0.26-1.22)$ & 0.143 & & \\
\hline \multicolumn{5}{|l|}{ Vascular invasion } \\
\hline No & Ref. & & Ref. & \\
\hline Yes & $5.13(1.41-18.63)$ & 0.013 & $3.22(0.79-13.16)$ & 0.104 \\
\hline \multicolumn{5}{|l|}{ Prior treatment } \\
\hline No & Ref. & & & \\
\hline Yes & $0.54(0.20-1.46)$ & 0.224 & & \\
\hline Surgical resection & $0.51(0.21-1.22)$ & 0.129 & & \\
\hline TACE & $0.57(0.26-1.28)$ & 0.176 & & \\
\hline RFA/PEI & $0.83(0.31-2.23)$ & 0.715 & & \\
\hline TKIs & $0.59(0.25-1.37)$ & 0.218 & & \\
\hline \multicolumn{5}{|c|}{ PD-1 inhibitors combined with TKIs } \\
\hline No & Ref. & & & \\
\hline Yes & $0.75(0.34-1.66)$ & 0.480 & & \\
\hline \multicolumn{5}{|l|}{ Overall response } \\
\hline No & Ref. & & & \\
\hline Yes & $0.31(0.04-2.37)$ & 0.259 & & \\
\hline \multicolumn{5}{|l|}{ Vascular-specific response } \\
\hline No & Ref. & & & \\
\hline Yes & $0.78(0.25-2.47)$ & 0.671 & & \\
\hline
\end{tabular}

The overall or organ-specific response was based on the combined number of patients with complete response and partial response. ECOG, Eastern Cooperative Oncology Group performance status; CLIP, Cancer of the Liver Italian Program scoring system; TACE, transcatheter arterial chemoembolization; RFA, radiofrequency ablation; PEI, percutaneous ethanol injection; TKI, tyrosine kinase inhibitor; PD-1 inhibitors, programmed cell death protein-1 inhibitors; Ref., reference level. ${ }^{\text {a }}$ The Cox proportional hazards model.

studies with a large number of patients are anticipated to elucidate this aspect.

This study has some limitations. First, it was conducted in a single center with a relatively small sample. Future large and multicenter cohort studies with longer followup periods are necessary to demonstrate the efficacy of
PD-1 inhibitors. Second, the current study focused on the radiographic assessment of the treatment response; additional information on potential predictive biomarkers for treatment response was not assessed. The identification of clinical features and biomarkers would allow for better patient selection for individual treatments [27]. 
Third, the mechanisms underlying the antithrombotic effect of immunotherapy warrant further investigation. Finally, the efficacy was similar between patients who received PD-1 inhibitors alone compared to those in whom PD-1 inhibitors were combined with TKIs. Preliminary data of clinical trials testing the combination of lenvatinib plus pembrolizumab showed an encouraging and attractive efficacy [2]. The patients in our study were heterogeneous with regard to regimens of TKIs or ICIs and prior systemic therapy. This strategy needs prospective evaluation in a more homogeneous and large-scale study.

In summary, this study showed that the tumor response to ICIs with PD-1 inhibitors varied among different organs in advanced HCC patients. The magnitude of the treatment response is significantly more intense in vascular invasion, and vascular response is also an independent prognostic factor relative to PFS. Future strategies should consider the role of added local therapy or combination therapies for oligoprogression in these organs for patients with otherwise sustained treatment benefit.

\section{Acknowledgments}

We thank Associate Prof. Sheng-Hsiang Lin and Chih-Hui Hsu for providing statistical consulting services from the Biostatistics Consulting Center, National Cheng Kung University Hospital. The authors also thank Enago (www.Enago.tw) for providing professional language editing and proofreading.

\section{Statement of Ethics}

Approval for this study was obtained from the Institutional Review Board of the National Cheng Kung University Hospital (AEX-108-019). This study was performed in accordance with the ethical principles of the Declaration of Helsinki by the World Medical Association. All patients provided written informed consent before inclusion in the study.

\section{Disclosure Statement}

The authors have no conflicts of interest to declare.

\section{Funding Sources}

This research did not receive any specific grants from funding agencies in the public, commercial, or not-for-profit sectors.

\section{Author Contributions}

H.-Y. Kuo: patient enrollment, data analysis, and editing. N.-J. Chiang: editing. C.-H. Chuang and C.-Y. Chen: patient enrollment. I.-C. Wu: statistical analysis. T.-T. Chang: supervision and editing. H.-M. Tsai: supervision and radiology review. Y.-J. Lin: project administration, supervision, and editing.

\section{References}

1 Vogel A, Cervantes A, Chau I, Daniele B, Llovet J, Meyer T, et al.; ESMO Guidelines Committee. Hepatocellular carcinoma: ESMO Clinical Practice Guidelines for diagnosis, treatment and follow-up. Ann Oncol. 2018 Oct;29 Suppl 4:iv238-55.

2 Ikeda M, Sung MW, Kudo M, Kobayashi M, Baron AD, Finn RS, et al. A phase $1 \mathrm{~b}$ trial of lenvatinib (LEN) plus pembrolizumab (PEM) in patients (pts) with unresectable hepatocellular carcinoma (uHCC). J Clin Oncol. 2018 May;36(Suppl 15):4076.

3 Cheng AL, Qin S, Ikeda M, Galle P, Ducreux M, Zhu A, et al. LBA3IMbrave150: Efficacy and safety results from a ph III study evaluating atezolizumab (atezo) + bevacizumab (bev) vs sorafenib (Sor) as first treatment (tx) for patients (pts) with unresectable hepatocellular carcinoma (HCC). Ann Oncol. 2019;30(Suppl 9).

4 Sharma P, Allison JP. The future of immune checkpoint therapy. Science. 2015 Apr; 348(6230):56-61.

5 Tumeh PC, Hellmann MD, Hamid O, Tsai KK, Loo KL, Gubens MA, et al. Liver metastasis and treatment outcome with Anti-PD-1 monoclonal antibody in patients with melanoma and NSCLC. Cancer Immunol Res. 2017 May;5(5):417-24.

6 Lin YY, Tan CT, Chen CW, Ou DL, Cheng $\mathrm{AL}$, Hsu C. Immunomodulatory effects of current targeted therapies on hepatocellular carcinoma: implication for the future of immunotherapy. Semin Liver Dis. 2018 Nov; 38(4):379-88.

7 Chan SL, Chong CC, Chan AW, Poon DM, Chok KS. Management of hepatocellular carcinoma with portal vein tumor thrombosis: review and update at 2016. World J Gastroenterol. 2016 Aug;22(32):7289-300.

8 Woo HY, Heo J. New perspectives on the management of hepatocellular carcinoma with portal vein thrombosis. Clin Mol Hepatol. 2015 Jun;21(2):115-21.

9 Yin J, Bo WT, Sun J, Xiang X, Lang JY, Zhong $\mathrm{JH}$, et al. New evidence and perspectives on the management of hepatocellular carcinoma with portal vein tumor thrombus. J Clin Transl Hepatol. 2017 Jun;5(2):169-76.

10 Rossi S, Ghittoni G, Ravetta V, Torello Viera F, Rosa L, Serassi M, et al. Contrast-enhanced ultrasonography and spiral computed tomography in the detection and characterization of portal vein thrombosis complicating hepatocellular carcinoma. Eur Radiol. 2008 Aug; 18(8):1749-56.

11 Eisenhauer EA, Therasse P, Bogaerts J, Schwartz LH, Sargent D, Ford R, et al. New response evaluation criteria in solid tumours: revised RECIST guideline (version 1.1). Eur J Cancer. 2009 Jan;45(2):228-47.
12 Lencioni R, Llovet JM. Modified RECIST (mRECIST) assessment for hepatocellular carcinoma. Semin Liver Dis. 2010 Feb;30(1):52-60.

13 Schmid S, Diem S, Li Q, Krapf M, Flatz L, Leschka $S$, et al. Organ-specific response to nivolumab in patients with non-small cell lung cancer (NSCLC). Cancer Immunol Immunother. 2018 Dec;67(12):1825-32.

14 Yu JI, Park JW, Park HC, Yoon SM, Lim DH, Lee $\mathrm{JH}$, et al. Clinical impact of combined transarterial chemoembolization and radiotherapy for advanced hepatocellular carcinoma with portal vein tumor thrombosis: an external validation study. Radiother Oncol. 2016 Feb;118(2):408-15.

15 Finn R, Ryoo BY, Merle P, Kudo M, Bouattour M, Lim HY, et al. Results of KEYNOTE-240: phase 3 study of pembrolizumab (Pembro) vs best supportive care (BSC) for second line therapy in advanced hepatocellular carcinoma (HCC). J Clin Oncol. 2019 Feb; 37(Suppl 15):4004.

16 Yau T, Park JW, Finn RS, Cheng A-L, Mathurin P, Edeline J, et al. LBA38_PRCheckMate 459: A randomized, multi-center phase III study of nivolumab (NIVO) vs sorafenib (SOR) as first-line (1L) treatment in patients (pts) with advanced hepatocellular carcinoma (aHCC). Ann Oncol. 2019 Sep; 30(Suppl 5). 
17 Scheiner B, Kirstein MM, Hucke F, Finkelmeier F, Schulze K, von Felden J, et al. Programmed cell death protein-1 (PD-1)-targeted immunotherapy in advanced hepatocellular carcinoma: efficacy and safety data from an international multicentre real-world cohort. Aliment Pharmacol Ther. 2019 May; 49(10):1323-33.

18 Llovet JM, Ricci S, Mazzaferro V, Hilgard P, Gane E, Blanc JF, et al.; SHARP Investigators Study Group. Sorafenib in advanced hepatocellular carcinoma. N Engl J Med. 2008 Jul; 359(4):378-90.

19 Cheng AL, Kang YK, Chen Z, Tsao CJ, Qin S, Kim JS, et al. Efficacy and safety of sorafenib in patients in the Asia-Pacific region with advanced hepatocellular carcinoma: a phase III randomised, double-blind, placebo-controlled trial. Lancet Oncol. 2009 Jan;10(1):25-34.
20 Chen DS, Mellman I. Elements of cancer immunity and the cancer-immune set point. Nature. 2017 Jan;541(7637):321-30.

21 Lu LC, Hsu C, Shao YY, Chao Y, Yen CJ, Shih IL, et al. Abstract 3627: organ-specific differential responses to immune checkpoint inhibitors in patients with advanced hepatocellular carcinoma. Cancer Res. 2018 Jul;78(13): 3627.

22 Bruix J, Cheng AL, Meinhardt G, Nakajima K, De Sanctis Y, Llovet J. Prognostic factors and predictors of sorafenib benefit in patients with hepatocellular carcinoma: analysis of two phase III studies. J Hepatol. 2017 Nov; 67(5):999-1008.
23 Jeong SW, Jang JY, Shim KY, Lee SH, Kim SG, Cha SW, et al. Practical effect of sorafenib monotherapy on advanced hepatocellular carcinoma and portal vein tumor thrombosis. Gut Liver. 2013 Nov;7(6):696-703.

24 Sharma P, Allison JP. Immune checkpoint targeting in cancer therapy: toward combination strategies with curative potential. Cell. 2015 Apr;161(2):205-14.

25 Kudo M. Combination cancer immunotherapy in hepatocellular carcinoma. Liver Cancer. 2018 Mar;7(1):20-7.

26 Iñarrairaegui M, Melero I, Sangro B. Immunotherapy of hepatocellular carcinoma: facts and hopes. Clin Cancer Res. 2018 Apr;24(7): 1518-24.

27 Patel SP, Kurzrock R. PD-L1 Expression as a predictive biomarker in cancer immunotherapy. Mol Cancer Ther. 2015 Apr;14(4):847-56. 